Ambient Science, 2016: Vol. 03(Sp1); 74-79

DOI:10.21276/ambi.2016.03.sp1.ra12

ambient

SCIENCE

Vol. 3(Sp1):74-79

Year 2016

\title{
Personal and Social Predictors about Safe Sexual Behavior in Patients with Immune Deficiency Virus in Ahwaz, Iran
}

\section{Shirin Hasanpoor', Soheyla Bani', Mojgan Mirghafourvand', Mansoureh Fakhouri' ${ }^{2 *}$}

'Department of Nursing and Midwifery, Tabriz University of Medical Sciences, Tabriz, Iran.

${ }^{2}$ Department of Nursing and Midwifery, Student Research Committee Tabriz University of Medical Sciences, Tabriz, Iran

Study Area: Ahwaz, Iran

Coordinate: $31^{\circ} 19^{\prime} 13^{\prime \prime} \mathrm{N} ; 48^{\circ} 40^{\prime} 09^{\prime \prime} \mathrm{E}$

Key words: HIV patients, Acquired immune deficiency syndrome, Social awareness, Safe sex

\section{Abstract}

Socio-demographic predictors about safe sex behaviours in individual suffering from immune deficiency virus (HIV) had been tried to understand in this cross-sectional study. It was conducted on 120 individuals having immune deficiency virus (HIV). Collection of the data were based on socio-demographic and a safe sex behaviour questionnaire. To determine the socio-demographic the general linear model was used. Result revealed mean (SD) of the total score of safe sexual behaviour among men and women was 66.5 (13.1), 62.2 (13.0) respectively and (Score limit: 0-100). Status of sexual partners, unprotected vaginal sex, drugs and alcohols, as well as employment status, were considered as predictors of safe sex behaviours. About 50 percent of the participants pose unsafe sexual practices, thus, it is advisable that the health promotion programs and HIV prevention should implement in various groups of the society.

condition, they can play an important role in HIV prevention (Allen et al., 2011). Although, unsafe sexual practices are done with people who are most likely HIV positive themselves, but an alarming rate of these practices are done with HIV-negative people or people who have an Unknown status of HIV / AIDS (Kalichman et al., 2006).

Success in AIDS treatment by antiretroviral drugs (HAART) increased the span and life quality of HIVpositive patients, however, the increment of their life span let to continuing their sexual relations due to which the chances of unsafe sexual practices also increase among HIV-positive people. Thus, the number of registered cases related to sexual transmission of HIV are continuously increasing (Yaya et al., 2014). Nevertheless, the drug injection consumers are also sexually active and their sexual practices are also unprotected (Wood et al., 2013). High-risk sexual practices are not rare among youths and use of Amphetamine stimulants is increasing and their effects on risky sexual practices are also alarming (Kogan et al., 2010). Registered cases of HIV positive in Iran between the year 1986 to 2012 show that out of all transmission of HIV through sexual practices and injection of drugs, $13.9 \%$ percent of total transmission were through risky sexual practices, and $67.2 \%$ percent were transmitted through drug injections which when practices after conducting test and being aware of their 
compared to collected data during the period of March 2oth,2012 to March 2oth, 2013, transmission of HIV through sexual practices increased to $36.8 \%$ percent and through drug injection in the same period of time were $45.5 \%$ percent (UNIAIDS, 2016). The spread of HIV through sexual practices is $34 \%$ percent among people had sex with opposite sex and $10 \%$ percent among people who had sexual practices with same sex while transmission through drug injection was reported $56 \%$ percent (Nasirian et al., 2012). Estimating the increasing rate of risky sexual practices in Iran specially among injecting and non-injecting drug users, prevention of risky sexual practices and increasing safe sexual practices in people affected by HIV/AIDS became one of the fundamental priorities in prevention and decreasing rate of HIV transmission in Iran (UNAIDS,2015; Sajadi et al., 2013; Haghdoost et al., 2012). Some studies were conducted in high-risk and at-risk of HIV/AIDS groups which show the existence of a noticeable rate of unsafe sexual practices and drug injection among younger generations. (Khajehkazemi et al., 2013; Nasirian et al., 2012). Also conducted some study on obstacles of using HIV/AIDS prevention tools through sexual practices such as condom (Langeroudi et al., 2014; Fallahi et al., 2012; Lotfi et al., 2012). Although prevention plans and statistical data with the aim of recognising HIV negative people has almost been done but there were lesser studies on recognising primary sources of HIV transmission which are in fact HIV/AIDS infected people (Kalichman et al., 2001). Also, HIV prevention activities and basic studies of HIV are some of the fundamental priorities of research in this field in Iran (Haghdoost et al., 2012). In fact, few studies have been conducted in this subject area so far particularly on knowledge of safe sex behaviour among HIV-positive people who are the primary source of infection and provide assistance in designing interventions intend to promote sexual health in this group. The present study aimed to determine the predictors of safe sexual practices in HIV-positive people and status of sexual behaviours in HIV-positive maleand female.

\section{Methodology:}

Subject selection: total 120 people; 89 male and 31 female who are HIV positive in the time period of October of 2013 to March of 2014 were selected as a subject. Patients were registered in two behavioural disorders consultation centres of west and east of Ahvaz city.

Sampling method in this study was the purposive type. To accomplish this task, information about this study, its goals, and method of implementing this research was provided to all the patients who had an active medical record in the respective consultation centres where their HIV serological tests were reported as positive and they visit those centres on regular basis. Further, participants were evaluated on the basis of their basic demographic data and enter-exit factors. Properly written consent from the patients about participating in the research was collected from them.

Inclusion criteria: HIV-positive male and female (based on the medical records in two behavioral disorders consultation centers), having at least 18 years old, being sexually active based on the declarations of patients (sexual activity means having vaginal, rectal or oral sexual practices at least once during the last six months), being literate, and having desire to participate in the study.

Data Collection: data collection tools included the socio-demographic questionnaire, and Safe Sexual Behavior Questionnaire (SSBQ). Socio-demographic questionnaire was based on the age, sex, income, education, race, marital status and the job profile which were filled up by the patients. Moreover with demographic factors some related factors regarding disorder (disorder stage, the number of years while diagnosed) and background of high-risk sexual behaviour which included the number of sexual partners, unprotected rectal, vaginal, and oral sexual practices were also mentioned in the same questionnaire. Participant were also asked to answer whether they had any other transmitted sexual infections rather than HIV or not. Whether they had received money for sexual practices or not. Whether they were having any history of drug injection, any drug, alcohol, methamphetamine and cocaine usage or not. And in case of the male, whether do they had sexual practice with male or not. Participants have also reported the number of their sexual partners, HIV status of their partner (includes HIV positive, HIV Negative, unknown status of HIV, or any other state).

Survey Instrument: SSBQ is a 24-question instrument that is designed by Dilario (1992) for measuring methods of reducing exposure and transmission of HIV. This questionnaire includes 25 questions that measure 5 following factors: 1- condom use 2- avoiding body fluids 3avoiding high-risk sexual practices $4^{-}$avoiding sexual intercourse with same sex or rectal practices, and 5Assertiveness skills (self-efficacy). The scale of this questionnaire is based on four-item Likert scale includes never, sometimes, often and always which evaluated with numbers 1 to 4 respectively.

To determine the scientific validity of this instrument, first validity of translation assessed with the forward and backwards translation method, then its contents have been investigated and evaluated by a group of 10 professors of faculties of Nursing and Midwifery University of Tabriz and after collecting regarded comments, suggested changes are done. Population sample was all the people having the clinical file in west and east centres of behavioural disorder consultation.

Analysis Procedure: analysis of data was done with SPSS 
statistical software. To describe demographic data, health, sexual behaviours, drug use, and total scale of high-risk sexual behaviours and safe intercourse descriptive statistical method including frequency, percentage, mean, and the standard deviation was used. To determine the relationship between demographic characteristics and the total score of the first safe sexual behaviour bivariate tests including t-test, and ANOVA were used. Then to control the confounding factors, all variables which had $\mathrm{p}<$ o.05 were entered into the general linear model.

\section{Resultsand Discussions:}

In this study, among 120 participants $74 \%$ percent of the participants were male and 25.5 percent were female. About half of the participants (50.8\%) were in the age group of 26 to 35 years old. The level of education of more than one-third of the participants $(35.8 \%)$ was primary school, 56 people $(46.7 \%)$ were married. About half of the patients $(45.8 \%)$ were Arabians and the remaining were Persians. In this study, out of 89 male participated, nearly half $(47.1 \%)$ were unemployed and out of 31 female participating in the study, more than three-quarters (77.4\%) were housewives (Table-1).

About half of the participants (51.7\%) stated that they have no income. About $\mathbf{1 2 . 5} \%$ of the participants had approximately one year past their diagnosis, and about half of them passed two to five years of diagnosis their illness. In relation to health and disease status, about half of the population did not require medical treatment and were not in the active phase of the illness. About one-third 35 percent are receiving antiretroviral drugs (ARVs), and 11.7 percent of the population despite having AIDS and the need to take ARVs did not take their medications (Table-1). In conjunction with the risk factors for HIV, less than half of the participants; $45 \%$ were injecting drug users in the past. About $3.3 \%$ of the participant transmitted infection from sex, sex for money and had male-male relationships. About a quarter $26.7 \%$ were women who were HIV infected by their husbands and had none of the risks of HIV. Further, about one-fifth; $19.4 \%$ of patients had more than one of the risk factors of HIV (Table-1). About half of the participants, $49.1 \%$ had two or more sexual partners, $46.7 \%$ of participants had sexual partners with unknown HIV status. Unprotected vaginal (about 87.5\%), rectal 43.3\% and oral $58.3 \%$ sexual practices were reported in population. History of drug use $71.7 \%$ and currently using drugs is $43.3 \%$ of the whole population. More than half $58.3 \%$ also had a prison record (Tableı).

The mean (SD) of safe sexual behaviour in men was 66.5 (13.1) in an attainable score of o to 100. The average score of sexual behaviour in women was 62.2 (13) in an attainable score of o to 100.

Based on the general linear model, there is a significant relationship between total score of safe sexual behaviour with unprotected vaginal sex, currently using drugs, the HIV status of sexual partners (with unknown status and negative) as well as participant's job $(\mathrm{p}<0.05)$ (Table-2).

Based on bivariate tests, there is a significant relationship between an overall score of safe sexual behaviour with the job, history of high-risk behaviours, sexual HIV status of partners, the number of sexual partners, unprotected vaginal intercourse, unprotected rectal practices, unprotected oral practices, disease status and currently using drugs.

Safe sexual behaviour score in the study was moderate to high. A significant positive correlation between safe sexual behaviours and other variables such as job, sexual partners, unprotected sexual practice (vaginal), and currently being a drug user has been revealed.

The average score of safe sexual behaviour was moderate to high. In a study in Uganda on HIV-positive people, the overall spread of safe sexual behaviours was $54.1 \%$, and there wasn't a significant difference between female $(56.3 \%)$ and male $(51.3 \%)(\mathrm{p}<0.131)$ (Bajunirwe et al., 2013). In a study, $60 \%$ of HIV positive that take ARTs revealed safe sexual behaviours and 34\% had high-risk sexual behaviours (Yaya et al., 2014). Stein et al. (2010) showed that a very few number of male participants had safe sex behaviours. According to Hajizadeh et al. (2012) that conducted on high-risk behaviours of HIV-positive people in Iran showed that $49.6 \%$ of males and $15.6 \%$ of females have had high-risk sexual behaviours.

In the current study, out of all participants who had unprotected vaginal sexual practices, the score of safe sexual behaviour was also lesser. There is a significant relationship between unprotected vaginal intercourse and high sexual activeness (Crosby et al., 2014). Two different studies conducted in 2003 and 2014 that almost $20 \%$ of participants have had unprotected vaginal intercourse and this has a signif icant relation with youth, income, sexual practices with non-spouse partners and lack of using other prevention tools (Grulich et al., 2003; deVisser etal., 2014).

In the current study, HIV status of the sexual partner is one of the other predictor factors of safe search behaviour. In such that HIV/AIDS positive individuals that having HIV-negative sexual partners had higher safe sexual behaviours compared to an HIV positive individual who had sexual partners with positive HIV/AIDS, unknown HIV/AIDS status, or any other status of HIV. One study in Uganda suggested that negativity of HIV/AIDS status of the sexual partner of the HIV-positive individual is one of the predictors of safe sexual behaviour (Musinguzi et al., 2014). In an another study in Uganda stated that HIV status of sexual partner had a significant relationship with high-risk behaviour; i.e. HIV-positive individual who was having a known HIV status partner (negative of positive) compared to people who had the 
Table 1.Demographic, health, sexual behavior and drug use in the past 6 months in women and men, HIV / AIDS

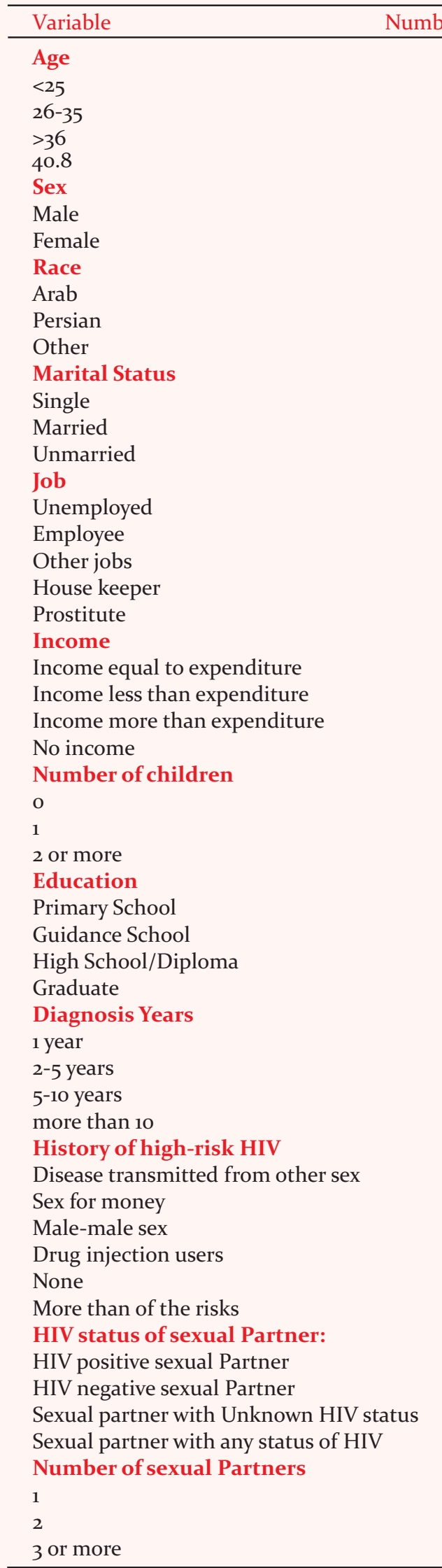

Ambient Science (2016)

$13 \quad 10.8$

$34 \quad 28.3$

$35 \quad 25$

$61 \quad 50.8$

$4 \quad 3.3$

$\begin{array}{ll}10 & 8.3 \\ 61 & 50.8 \\ 49 & \end{array}$

$\begin{array}{ll}89 & 74.2 \\ 31 & 25.8 \\ & \\ 55 & 45.8 \\ 54 & 45 \\ 11 & 9.2\end{array}$

$\begin{array}{ll}54 & 45 \\ 56 & 46\end{array}$

$10 \quad 8.3$

$42 \quad 35$

$34 \quad 28.3$

2420

$7 \quad 5.8$

$19 \quad 15.8$

$5 \quad 4.2$

$62 \quad 51.7$

$18 \quad 27 \cdot 3$

$22 \quad 33 \cdot 3$

$26 \quad 39.4$

$43 \quad 35.8$

$37 \quad 30.8$

$30 \quad 25$

$10 \quad 8.3$

$15 \quad 35.8$

$\begin{array}{ll}66 & 30.8\end{array}$

$4 \quad 8.3$

$4 \quad 3 \cdot 3$

$4 \quad 3.3$

$4 \quad 3 \cdot 3$

$54 \quad 45$

$32 \quad 26.7$

$22 \quad 19.4$

$\begin{array}{ll}32 & 26.7\end{array}$

$25 \quad 20.8$

$\begin{array}{ll}56 & 46.7\end{array}$

$7 \quad 5.8$

$55 \quad 45.8$
Unprotected Vaginal intercourse:

YES

YES

$105 \quad 87.5$

Oral unprotected sexual practice:

YES

$52 \quad 43 \cdot 3$

Disease Status:

HIV positive $\quad 64 \quad 53.3$

AIDS $\quad 14 \quad 11.7$

Retroviral drugs intake $\quad 42 \quad 35$

History of drug use in the past:

$\begin{array}{lll}\text { YES } & 86 & 71.7\end{array}$

Current drug use:

$\begin{array}{lll}\text { YES } & 28 & 43.3\end{array}$

Prison Record:

YES

$\begin{array}{ll}70 & 58.3\end{array}$

Table-2: Relationship of demographic, health, sexual behavior, drug-use and overall score of high-risk sexual behaviour with safe sex in the past 6 months in men and women infected by HIV/AIDS based on the general linear model

\begin{tabular}{llll}
\hline Variable & $\mathrm{B}$ & $(\mathrm{CI} 95 \%)$ & p-value \\
\hline $\begin{array}{llll}\text { History of high-risk HIV behavior } \\
\text { YES (Ref.) }\end{array}$ & & $\mathrm{o}$ & \\
NO & 2.4 & $(-2.2$ to 7$)$ & 0.305 \\
HIV status of sexual partner: & & & \\
With any condition of HIV (Ref.) & & $\mathrm{o}$ & \\
HIV positive & 1.3 & $(-6.3$ to 9) & 0.726 \\
HIV negative & 11.7 & $(3.5$ to 19.8) & 0.005 \\
HIV unknown & 8.5 & $(1.2$ to 15.8) & 0.023 \\
JOB: & & & \\
Prostitute (reference) & & 0 & \\
Unemployed & 6.3 & $(-1.6$ to 14.3) & 0.118 \\
Employee & 12 & $(2.3$ to 21.6) & 0.016 \\
Other jobs & 6.9 & $(-1.5$ to 15.3) & 0.107 \\
House Keeper & 4.3 & $(-5.3$ to 14) & 0.378
\end{tabular}

Type of sexual behavior:

Homosexual \& straight sexual (Ref.) $\quad 0$

non-homosexua $\quad 6.6 \quad(-1.8$ to 9.1$) \quad 0.185$

Unprotected vaginal sexual practice

NO (reference) o

YES $\quad-21 \quad(-14.6$ to -3.7$) \quad 0.001$

Unprotected rectal sexual practice

NO (reference) 0

$\begin{array}{llll}\text { YES } & -2.6 & (-6.8 \text { to }-2.5) & 0.257\end{array}$

Unprotected oral sexual practice

NO (reference)

YES $\quad-1.9 \quad(-6.4$ to -2.5$) \quad 0.386$

Currently a drug user

NO (Ref.)

YES

$11.4 \quad(7.01$ to 15.78$)<0.001$

Number of sexual partners

3 or more (Ref.)

one

Two

Status of Disease

Retroviral drug intake (Ref.)

HIV Positive

AIDS

$$
\begin{array}{llll} 
& 0 & \\
2.5 & (-1.59 \text { to } 6.7) & 0.225 \\
4.9 & (-4 \text { to } 14) & 0.227 \\
& & \\
& 0 & \\
-1.9 & (-5.5 \text { to } 1.6) & 0.278 \\
-2.1 & (-7.5 \text { to } 3.3) & 0.447
\end{array}
$$


unknown status of the partner had higher safe sexual practices (Bajunirwe et al., 2013). Findings of both the mentioned studies are conf irmed in the current study.

One of the other predictors of safe sexual behaviours in the current study was alcohol and drug usage. People who were then using alcohol and drugs having lesser safe sexual behaviours compared to people who were not using any alcohol and drugs. Many studies mentioned the effect of alcohol and drug usage on safe sexual practices (Fallahi et al., 2012; Hojjat et al., 2012).

In a case study, it was noted that in addition to the relationship between personality structure of individual and risky sexual behaviour among HIV / AIDS positives, factors such as perceived responsibility and drug use were some other predictors of high-risk sexual behaviours (Shuper et al., 2014). In a study in the Iran, it was reported that possibility of high-risk sexual behaviours is related to addiction to drugs and usage of alcohol (Hajizadeh et al., 2013). Hojjat (2011) in his research reported that there is a relationship between alcohol usages in individuals under methadone medication with increasing high-risk sexual behaviours. Also, high-risk sexual behaviours had a significant relationship with drug usage. Nazarius et al. (2012) reported that there was a highly significant relationship between alcohol consumption, having multiple sexual partners and unprotected sexual practices.

\section{Conclusion:}

In our study, the condition of safe sexual behaviours in HIV/AIDS positive individuals was found to be on the higher side than average and factors such as HIV-positive sexual partner, unprotected vaginal intercourse, alcohol and drug usage, and also job condition were significantly related to safe sexual behaviours. We found that about half of the participants did not have safe sexual behaviours, regarding this fact that safe sexual behaviours in HIV/AIDS infected people would have a signif icant role in decreasing the spread of HIV among healthy individuals and also decreasing cases of transmitted infection of HIV type or different strains of HIV in infected people. These findings show the necessity of more efforts to promote HIV prevention programs in Iran. Besides the HIV prevention programs and promoting social health strategies, more stress must be given in finding HIVinfected people in the deeper insight into different groups of society; especially within them who are sexually active and also HIV-positive sexually active patients.

Limitations and Future Research Directions: this study has some limitationsin itseld. For example the current population sample cannot represent the whole country; because the volume of the population sample is 120 individuals and the number of female participants is far lesser than males. The second limitation is that high- risk sexual behaviours are self-reported and there is the possibility that because of social biases some cases were not reported at all. It is better if the number of female participants increases in the future researchers.

Acknowledgements:

We should thank and appreciate the efforts of Center of Consultation of Behavioral Disorders of Ahvaz for their cooperating in sampling and issuing necessary recommendation, Dr. Sayed Mohammad Alavi, infectious disease specialist and Dr. Shokr-Allah Salmanzadeh, infectious disease specialist and director of Health center of Khuzestan province for his motivations and support in this study, Dr. Farashahi infectious disease specialist regarding providing necessary scientific resources and Dr. Azadeh Mehrpouyan for her suggestions and editing article derived from master degree thesis.

\section{References:}

Allen, C., Mbonye, M., Seeley, J., Birungi, J., Wolff, B., Coutinho, A., Jaffarf, Sh. (2011): ABC for people with HIV: responses to sexual behavior recommendations among people receiving antiretroviral therapy in Jinja, Uganda. Cult Health Sex., 13(5):529-543.

Bajunirwe, F., Bangsberg, D. \& Sethi, A. (2013): Alcohol use and HIV serostatus of partner predict high-risk sexual behavior among patients receiving antiretroviral therapy in South Western Uganda. BMC Public Health, 13:430.

Crosby, R.A., Milhausen, R.R., Sanders, S., Graham, C.A. \& Yarber, W.L. (2014): Being drunk and high during sex is not associated with condom use behaviours: a study of high-risk young Black males. Sex Health, 11(1):84-86.

deVisser, R.O., Badcock, P.B., Rissel, C., Richters, J., Smith, A.M., Grulich, A.E. \& Simpson, J.M. (2014): Safer sex and condom use: findings from the Second Australian Study of Health and Relationships. Sex Health, 11(5):495-504.

Fallahi, H., Tavfian, S., Yaghmaie, F., Hajizadeh, E. (2012): Perceived barriers of condom use in people living with HIV/AIDS: a qualitative research. Payesh, 11(6):831-840

Faramarzi, H. \& Bagheri, P. (2012): Distribution of different kinds of sexual risk behaviors in patients referring to Counseling Center of Behavioral Disorders. Zahedan J. Res. Med. Sci., 14(2):105

Grulich, A.E., deVisser, R.O., Smith, A.M., Rissel, C.E. \& Richters, J. (2003): Sex in Australia: injecting and sexual risk behaviour in a representative sample of adults. Aust. N.Z.J. Public Health. 27(2):242-250.

Haghdoost, A., Sadeghi, M., Nasirian, M., Mirzazadeh, A. \& Navadeh, S. (2012): Research priorities in the field of HIV and AIDS in Iran. J. Res. Med. Sci., 17(5):481-486.

Hajizadeh, S.H., Nejat, S., Majdzadeh, S.R., Moharez, M., Setayesh, H.R. \& Gooya, M.M. (2013): High-risk behaviors of HIV positive individuals referred to Behavioral Counselling Centers of Universities of Medical Sciences in Tehran. I. School Publ. Health Inst. Pub. Health Res., 10(3):65-76

Hojjat, S.K., Tabatabaichehr, T., Mortazavi, H., Ebrahimi, E., Muradian, H. \& Jafari, J. (2012): Compare psychological profile of patients undergoing methadone treatment for drug dependent people in Bojnoord. J. North Khorasan Uni. Med.Sci., 4(4):553-561. 
Khajehkazemi, R., Osooli, M., Sajadi, L., Karamouzian, M., Sedaghat, A., Fahimfar, N., Safaie, A., Mostafavi, E. \& Haghdoost, A. (2013): HIV prevalence and risk behaviors among people who inject drugs in Iranthe 2010 National Surveillance Survey, Sex Transm Infect. DOI:10.1136/sextrans2013-051204

Kalichman, S.C., Rompa, D., Cage, M., DiFonzo, K., Simpson, D., Austin, J., Luke, W., Buckles, J., Kyomugisha, F., Benotsch, E., Pinkerton, S. \& Graham, J. (2001): Effectiveness of an intervention to reduce HIV transmission risks in HIVpositive people. Am. J. Prev. Med., 21(2):84-92.

Kalichman, S.C., Eaton, L., Cain, D., Cherry, C., Pope, H. \& Kalichman, M. (2006): HIV treatment beliefs and sexual transmission risk behaviors among HIV positive men and women. JBehav Med., 29(5):401-410.

Kogan, S., Brody, G., Chen, Y., Grange, C., Slater, L. \& DiClemente, R. (2010): Risk and Protective Factors for Unprotected Intercourse among Rural African American Young Adults. Pub. Health Rep., 125(5):709-717.

Langeroudi, M., Afarin, R., Movaghar, Sh., Ahmadi, D. \& Esmaeili, M. (2014): Barriers of condom use among female sex workers in Tehran, A qualitative study. LSchool Pub. Health Ins. Pub. Health Res., 2(1):25-37

Lotfi, R., Tehrani, R.F., Yaghmayee, F. \& Hajizadeh, E. (2012): Social and environmental barriers to condom use in women at risk for HIV / AIDS: A Qualitative Study. Payesh, 5(45):669-678

Musinguzi, G, Bwayo, D., Kiwanuka, N., Coutinho, S., Mukose, A., Kabanda, J., Sekabembe, L. \& Nuwaha, F. (2014): Sexual behavior among persons living with HIV in Uganda: implications for policy and practice. PloS One. 9(1):e85646.
(2012): Modeling of human immunodeficiency virus modes of transmission in Iran. J. Res. Health Sci., 12 (2):81-87.

Stein, J.A., RotheramBorus MJ, Swendeman D, Milburn NG. (2005): Predictors of sexual transmission risk behaviors among HIV-positive young men. AIDS Care, 17 (4):433442

Sajadi, L., Mirzazadeh, A., Navadeh, S., Osooli, M., Khajehkazemi, R., Gouya, M., Fahimfarm, N., Zamani, O. \& Haghdoost, A. (2013): HIV prevalence and related risk behaviours among female sex workers in Iran: results of the national. biobehavioural survey. Sex Transm Infect. 89(3):37-40.

Shuper, P.A., Joharchi, N. \& Rehm, J. (2014): Personality as a predictor of unprotected sexual behavior among people living with HIV/AIDS: a systematic review. AIDS Behav., 18(2):398-410.

Wood, J.R., Milhausen, R.R., Sales, J.M., Graham, C.A., Sanders, S.A., Diclemente, R.J. \& Wingood, G.M. (2013): Arousability as a predictor of sexual risk behaviours in African-American adolescent women. Sex Health, 10(2):160-165

UNIAIDS (2015): Islamic Republic of Iran AIDS Progress Report. (http://www.unaids.org/sites/default/files/country/docum ents/IRN_narrative_report_2015.pdf)

UNAIDS (2016): Fact sheet, November 2016 Available from: (http://www.unaids.org/en/resources/campaigns/HowAID Schangedeverything/factsheet)

Yaya, I., Saka, B., Landoh, D.E., Patchali, P.M., Makawa, M.S., Senanou, S., Idrissou, D., Lamboni, B. \& Pitche, P. (2014): Sexual risk behavior among people living with HIV and AIDS on antiretroviral therapy at the regional hospital of Sokodé, Togo. BMC Public Health, 14:636.

Nasirian, M., Doroudi, F., Gooya, M., Sedaghat, A. \& Haghdoost, A. 